\title{
SSBI Mitigation and the Kramers-Kronig Scheme in Single-Sideband Direct-Detection Transmission With Receiver-Based Electronic Dispersion Compensation
}

\author{
Zhe Li, Student Member, IEEE, M. Sezer Erkılınç, Member, IEEE, Kai Shi, Member, IEEE, \\ Eric Sillekens, Student Member, IEEE, Lidia Galdino, Member, IEEE, Benn C. Thomsen, Member, IEEE, \\ Polina Bayvel, Fellow, IEEE, and Robert I. Killey, Member, IEEE
}

\begin{abstract}
The performance of direct-detection transceivers employing electronic dispersion compensation combined with DSP-based receiver linearization techniques is assessed through experiments on a $4 \times 112 \mathrm{~Gb} / \mathrm{s}$ wavelength-division multiplexing direct-detection single-sideband 16 quadratic-amplitude modulation Nyquist-subcarrier-modulation system operating at a net optical information spectral density of $2.8 \mathrm{~b} / \mathrm{s} / \mathrm{Hz}$ in transmission over standard single mode fiber links of up to $240 \mathrm{~km}$. The experimental results indicate that systems with receiver-based dispersion compensation can achieve similar performance to those utilizing transmitter-based dispersion compensation, provided it is implemented together with an effective digital receiver linearization technique. The use of receiver-based compensation would simplify the operation of a fiber link since knowledge of the link dispersion is not required at the transmitter. The recently proposed Kramers-Kronig receiver scheme was found to be the best performing among the receiver linearization techniques assessed. To the best of our knowledge, this is the first experimental demonstration of the Kramers-Kronig scheme.
\end{abstract}

Index Terms-Beating interference cancellation, direct detection, Kramers-Kronig receiver, Nyquist subcarrier modulation, receiver-based electronic dispersion compensation, spectrallyefficient wavelength division multiplexing.

\section{INTRODUCTION}

D UE to their simplicity and relatively low cost, singlepolarization direct-detection (DD) transceivers may be an attractive technology for short and medium reach optical fiber transmission systems, for example in metropolitan, back-haul, access and inter-data center applications. In DD systems, singlesideband (SSB) quadrature amplitude modulation (QAM) subcarrier modulation (SCM) including orthogonal frequency division multiplexing (OFDM) and Nyquist subcarrier modu-

Manuscript received December 12, 2016; revised February 3, 2017; accepted March 9, 2017. Date of publication March 20, 2017; date of current version April 20, 2017. This work was supported in part by the EU ERA-NET+ project, in part by the U.K. EPSRC UNLOC EP/J017582/1 project, in part by the EU FP7 ASTRON project, and in part by the Semtech Corporation. (Corresponding author: Zhe Li.)

The authors are with the Optical Networks Group, Department of Electronic and Electrical Engineering, University College London, London WC1E 7JE, U.K. (e-mail: zhe.li@ee.ucl.ac.uk; m.erkilinc@ee.ucl.ac.uk; k.shi@ucl.ac.uk; e.sillekens@ee.ucl.ac.uk; 1.galdino@ee.ucl.ac.uk; b.thomsen@ucl.ac.uk; p.bayvel@ucl.ac.uk; r.killey@ucl.ac.uk).

Color versions of one or more of the figures in this paper are available online at http://ieeexplore.ieee.org.

Digital Object Identifier 10.1109/JLT.2017.2684298 lation (Nyquist-SCM) have shown strong potential as signaling schemes to achieve high information spectral density (ISD) $(\geq 2(\mathrm{~b} / \mathrm{s}) / \mathrm{Hz})$ [1]-[5]. For complexity and cost reasons, it is preferable to compensate the accumulated dispersion of the fiber link digitally, a technique referred to as electronic dispersion compensation (EDC), rather than by utilizing optical compensation methods such as dispersion compensating fiber (DCF). EDC can be performed at the transmitter (Tx-EDC) using the complex modulator, with good performance [6]. However, the nonlinearity introduced by square-law photodetection can significantly impair the performance of direct-detection systems with receiver-based EDC (Rx-EDC). One proposal to overcome this limitation is to use optical single-sideband signaling, with the aim of preserving the optical phase waveform of the signal in the electrical domain after detection, allowing the use of RxEDC. This approach was demonstrated with the SSB PAM-2 signal format in [7]. However, even in the case of SSB signaling, the system performance with Rx-EDC is limited by the nonlinear effect known as signal-signal beat interference (SSBI) caused by square-law detection. In SSB OFDM and NyquistSCM demonstrations to date, the dispersion is compensated by either a cyclic prefix (CP) to achieve dispersion tolerance [8]-[10] or by performing transmitter-based EDC (Tx-EDC) [4], [5]. However, the disadvantages include the reduction in the achievable spectral efficiency due to the $\mathrm{CP}$, or increased complexity of the system operation due to the required knowledge of the link's cumulative dispersion at the transmitter and hence the need for feedback from the receiver. Moreover, the increase of peak-to-average power ratio (PAPR) caused by Tx-EDC may lead to larger quantization noise from the digital-to-analog converters (DACs) and modulation nonlinearities. Thanks to the recent development of digital linearization techniques [9]-[18], the nonlinear SSBI distortion can be partially compensated or avoided. As suggested in [11], effective linearization techniques offer the possibility of performing the compensation of linear optical effects, such as dispersion, at the receiver with similar performance to systems with pre-compensation.

In this paper, we experimentally assess the performance of Rx- and Tx-EDC schemes, both in theory and experiments, in the presence of five recently proposed receiver-based digital linearization techniques, namely a single-stage linearization filter [10], a two-stage linearization filter [12], an iterative linearization filter [5], an SSBI estimation and cancellation technique [13] and the Kramers-Kronig scheme [11], [19]. The first four approaches treat the signal-signal beating terms as 


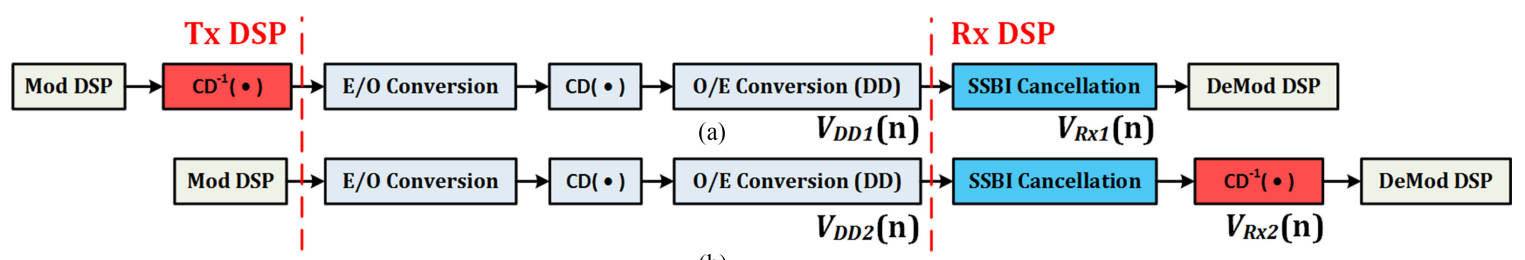

(b)

Fig. 1. Direct-detection system diagram with (a) Tx-EDC and (b) Rx-EDC combined with SSBI cancellation. Mod \& Demod DSP: Modulation and demodulation DSP for SSB Nyquist-SCM signal. O/E conversion consists of an optical band-pass filter followed by a single-ended photodiode.

a perturbation to the signal. The system compensation works by calculating these terms and subtracting them from the detected signal. In contrast, the latter approach (the Kramers Kronig scheme) reconstructs the optical phase of the signal from the detected amplitude waveform, making the assumption that it is a single-sideband and minimum phase signal. The experimental demonstrations were carried out using a $4 \times$ $112 \mathrm{~Gb} / \mathrm{s}$ spectrally-efficient (net ISD of $2.8(\mathrm{~b} / \mathrm{s}) / \mathrm{Hz}$ ) WDM DD SSB 16-QAM Nyquist-SCM system over transmission distances of $80 \mathrm{~km}, 160 \mathrm{~km}$ and $240 \mathrm{~km}$. The performance of both Tx- and Rx-EDC schemes were compared for the cases without and with the linearization techniques. Experimental results indicate that the difference in system performance with Tx- and Rx-EDC depends strongly on the performance of the linearization scheme being used, and that they can achieve similar performance provided an effective linearization technique is performed prior to dispersion compensation at the receiver. Moreover, to the best of our knowledge, this is the first reported experimental demonstration of the Kramers-Kronig algorithm, which we found provides superior performance compared to the other SSBI cancellation techniques.

\section{PRINCIPLE OF OPERATION}

In order to assess the effectiveness of Rx- and Tx-EDC combined with linearization techniques, the two DD systems shown in Fig. 1(a) and (b) were considered. For the first configuration (Fig. 1(a)), the SSB QAM Nyquist-SCM signal $E_{0}(n)$ is generated at the transmitter, following the modulation DSP. Tx-EDC, denoted as $\left(H_{C D}^{-1}(\bullet)\right)$, is performed by pre-distorting the signal to compensate the chromatic dispersion, as proposed in [6]. Following this, the optical carrier, $E_{\text {carrier }}$ is added during the electrical-to-optical conversion using an IQ-modulator. After the transmission and square-law detection, the normalized detected double-sideband (DSB) signal, $V_{D D}(n)$ can be written as:

$$
\begin{aligned}
V_{D D 1}(n) & =\left|H_{C D}\left(E_{\text {carrier }}+H_{C D}^{-1}\left(E_{0}(n)\right)\right)\right|^{2} \\
& =E_{\text {carrier }}^{2}+2 R e\left[E_{\text {carrier }} \cdot E_{0}(n)\right]+\left|E_{0}(n)\right|^{2},
\end{aligned}
$$

where $\operatorname{Re}[x]$ signifies the real part of $x$. In the RHS of (1), the first and second terms are the direct current (DC) and the desired carrier-signal beating products (CSBP), the third term is the signal-signal beating term. Assuming that the third term can be completely removed by performing SSBI compensation or through the use of the Kramers-Kronig scheme, the received signal prior to the demodulation DSP, $V_{R x 1}(n)$ only includes the DC and the desired CSBP terms and can be re-written as follows:

$$
V_{R x 1}(n) \approx E_{\text {carrier }}^{2}+2 R e\left[E_{\text {carrier }} \cdot E_{0}(n)\right] .
$$

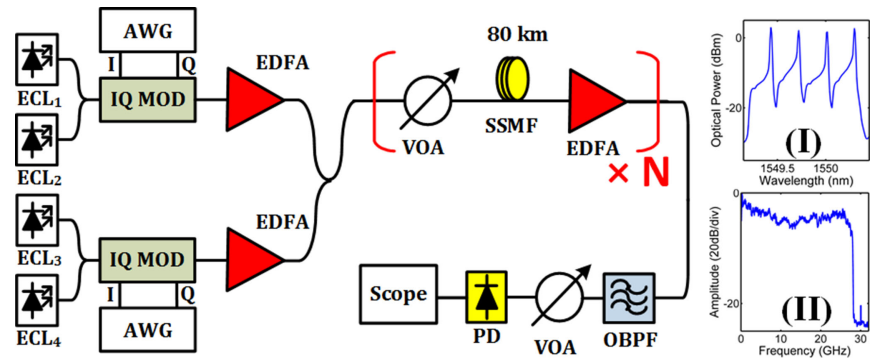

Fig. 2. Optical transmission experimental test-bed. Inset: (I) Experimental WDM and (II) received and digitized signal spectra. ECL: External cavity laser. PC: Polarization controller. AWG: Arbitrary waveform generator. EDFA: Erbium-doped fiber amplifier. VOA: Variable optical attenuator. SSMF: Standard single-mode fiber. OBPF: Optical band-pass filter. PD: Photodiode.

For the second configuration shown in Fig. 1(b), if Rx-EDC is used instead of Tx-EDC, the detected signal $V_{D D 2}(n)$ is given by:

$$
\begin{aligned}
V_{D D 2}(n)= & E_{\text {carrier }}^{2}+2 R e\left[E_{\text {carrier }} \cdot H_{C D}\left(E_{0}(n)\right)\right] \\
& +\left|H_{C D}\left(E_{0}(n)\right)\right|^{2}
\end{aligned}
$$

In contrast to (1), it can be seen that the second and third terms become the beating products between the dispersed signal with the optical carrier and with itself, respectively. If RxEDC, as proposed in [7], [21], is performed without linearizing the receiver, the frequency-dependent phase rotation of the SSBI terms due to dispersion, denoted as $H_{C D}^{-1}\left(\left|H_{C D}\left(E_{0}(n)\right)\right|^{2}\right)$, prevents the Rx-EDC from accurately recovering the undispersed signal. Therefore, the performance of Rx-EDC is significantly degraded in contrast to that of Tx-EDC. However, assuming that the third term can be largely removed by performing digital linearization and Rx-EDC is utillized afterwards, the signal before the demodulation DSP $V_{R x 2}(n)$ becomes:

$$
\begin{aligned}
V_{R x 2}(n) & \approx H_{C D}^{-1}\left(E_{\text {carrier }}^{2}+2 R e\left[E_{\text {carrier }} \cdot H_{C D}\left(E_{0}(n)\right)\right]\right) \\
& \approx E_{\text {carrier }}^{2}+2 R e\left[E_{\text {carrier }} \cdot E_{0}(n)\right] .
\end{aligned}
$$

By comparing (2) and (4), it can be seen that the RxEDC can achieve similar performance to Tx-EDC provided the SSBI term is removed. Therefore, it can be concluded that the performance of Rx-EDC depends on the effectiveness of the linearization achieved through the beating interference compensation scheme. It should be noted that, in this study, both Tx- and Rx-EDC is carried out by linear convolution with the inverse of the channel response.

\section{EXPERIMENTAL SETUP}

An optical transmission test-bed, shown in Fig. 2, was used to experimentally assess the performance of the transceiver de- 
signs. The odd and even channels were generated using two IQmodulators, which were driven by a pair of arbitrary waveform generators (AWGs), operating at $92 \mathrm{GSa} / \mathrm{s}$ sampling rate with $33 \mathrm{GHz} 3-\mathrm{dB}$ bandwidth. In the transmitter DSP, the signal waveforms of the $112 \mathrm{~Gb} / \mathrm{s}$ (symbol rate $\left(f_{s}\right)$ of $28 \mathrm{GBaud}$ ) SSB 16-QAM Nyquist-SCM signal were generated by applying a pair of root-raised-cosine (RRC) filters with a roll-off factor of 0.01 to in-phase (I) and quadrature (Q) components, and subsequently, the baseband complex signal was frequency up-converted to a subcarrier frequency of $14.28 \mathrm{GHz}$ (equal to $0.51 \times f_{s}$ ). Full details of Nyquist-SCM modulation and demodulation are given in [4]. Following the generation of modulated odd and even channels, centered at a wavelength of $1550 \mathrm{~nm}$, they were multiplexed to form four WDM channels with decorrelated neighboring channels and a channel spacing of $37.5 \mathrm{GHz}$, leading a gross information spectral density of $3.0(\mathrm{~b} / \mathrm{s}) / \mathrm{Hz}$. In the experiment, the optical carrier was generated by biasing the IQ-modulators above the null point and the biases were adjusted to achieve the optimum optical carrierto-signal power ratio (CSPR, the value of which is dependent on the optical signal-to-noise ratio (OSNR)), while the radio frequency (RF) voltage swing was kept constant (3.4V).

Transmission experiments were carried out using a straightline multiple span fiber link, with standard single-mode fiber (SSMF) spans of length $80 \mathrm{~km}$, and erbium-doped fiber amplifiers (EDFAs) with $5 \mathrm{~dB}$ noise figure. At the receiver, the channel of interest was demultiplexed using an optical band-pass filter with a 3-dB bandwidth of $31 \mathrm{GHz}$, and then detected using a single-ended PIN photodiode followed by digitization using a single ADC. In the receiver DSP (Rx DSP), the signal was initially resampled to 2 samples/symbol. The beating interference compensation or Kramers-Kronig scheme was performed digitally for receiver linearization followed by the Rx-EDC. In this work, five recently proposed receiver linearization techniques were tested. Following this, signal demodulation including frequency down-conversion and RRC matched filtering were carried out. The performance of the transceiver with Rx-EDC was compared with that using Tx-EDC in the case of no linearization and with different linearization techniques as described in the following section.

\section{EXPERIMENTAL RESULTS}

This section describes the WDM optical back-to-back and transmission performance of both EDC schemes combined with different linearization techniques, including the single-stage, two-stage, iterative linearization filters, SSBI estimation and cancellation technique, and the Kramers-Kronig (KK) scheme. Optical back-to-back performance was evaluated by performing amplified spontaneous emission (ASE) noise loading at the receiver, while transmission performance evaluation was carried out through transmission over distances of up to $240 \mathrm{~km}$. In order to achieve the optimum system performance, the CSPR value was swept from $2 \mathrm{~dB}$ to $16 \mathrm{~dB}$, and optimized at each OSNR value. This optimization is crucial to achieve the optimum performance due to the trade-off between the nonlinear and linear beating products [15].

For the purpose of assessing the WDM optical back-to-back performance, the BER curves versus OSNR at $0.1 \mathrm{~nm}$ resolution bandwidth are plotted in Fig. 3. The measurement of the signal power includes the optical carrier and the 16QAM-modulated sideband.

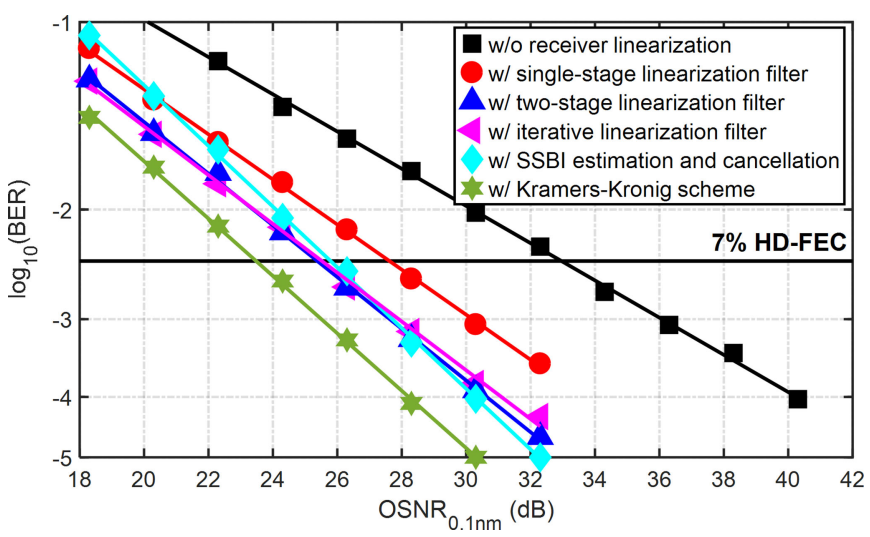

Fig. 3. BER vs OSNR without and with SSBI cancellation and KramersKronig scheme.

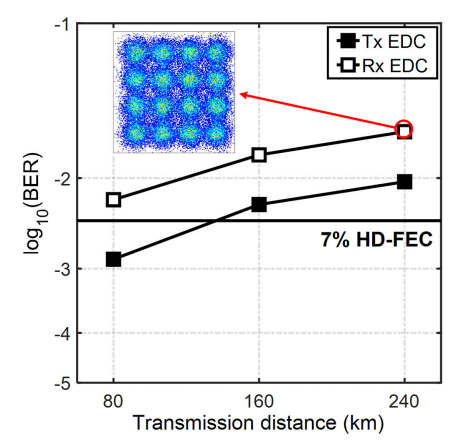

(a)

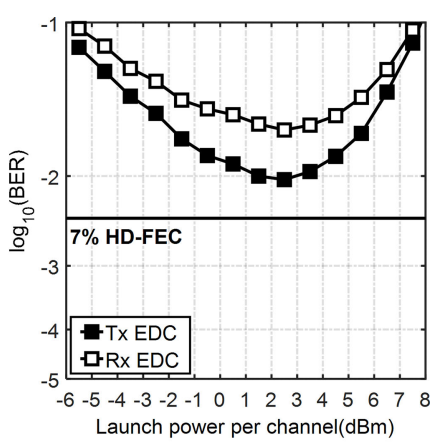

(b)
Fig. 4. (a) BER vs transmission distance with Tx-EDC and Rx-EDC without beating interference mitigation. Inset: Received constellation with Rx-EDC $(E V M=23.0 \%)$. (b) BER vs launch power per channel with Tx-EDC and Rx-EDC without beating interference mitigation.

\section{A. Without Receiver Linearization}

In back-to-back operation (Fig. 3), the required OSNR value at the hard-decision forward error correction (HD-FEC) threshold $\left(\mathrm{BER}=3.8 \times 10^{-3}\right)$ was found to be $32.9 \mathrm{~dB}$ without linearization. As discussed in Section II, if Rx-EDC is performed without compensating the beating interference, the introduced extra distortion prevents the receiver from recovering the dispersed signal. A comparison of the performance of RxEDC and Tx-EDC without beating interference compensation is plotted in Fig. 4. Significant performance differences can be observed between the Rx-EDC and Tx-EDC. As shown in Fig. 4(a), for transmission distances from $80 \mathrm{~km}$ to $240 \mathrm{~km}$, the BER ranged from $6.3 \times 10^{-3}$ to $2.4 \times 10^{-2}$ for Rx-EDC whereas much lower BER values were obtained with Tx-EDC (from $1.3 \times 10^{-3}$ to $9.3 \times 10^{-3}$ ), as predicted by the analysis in Section II: the nonlinear beating interference prevents the Rx-EDC from accurately recovering the undispersed signal. Fig. 4(b) shows a comparison of both EDC schemes through plots of BER versus optical launch power per channel.

\section{B. With Single-Stage Linearization Filter}

Fig. 5 shows the Rx DSP design using the single-stage linearization filter followed by Rx-EDC. In the single-stage linearization filter [10], a digital SSB signal is first generated from the detected signal using a sideband filter (SF), and an approximation of the waveform of the signal-signal beating products is calculated based on this SSB signal, which is then subtracted 


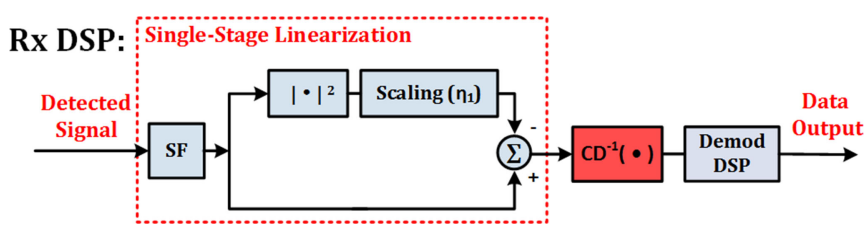

Fig. 5. Receiver DSP including single-stage linearization filter and Rx-EDC Demod DSP: Conventional demodulation DSP for SSB Nyquist-SCM signal. SF: Sideband filter.

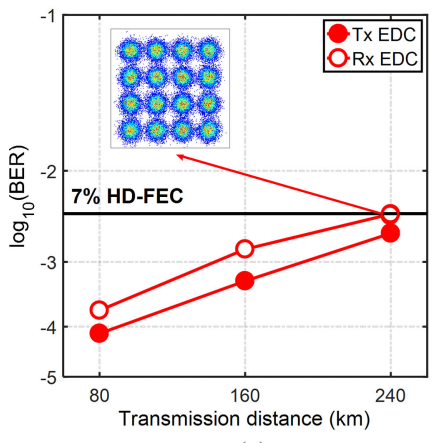

(a)

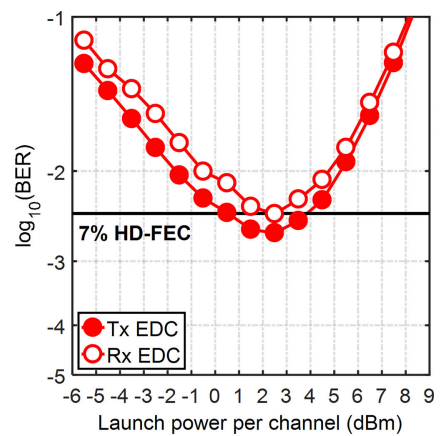

(b)
Fig. 6. (a) BER vs transmission distance with Tx-EDC and Rx-EDC using the single-stage linearization filter. Inset: Received constellation with Rx-EDC $(E V M=18.5 \%)$. (b) BER vs launch power per channel with Tx-EDC and $\mathrm{Rx}-\mathrm{EDC}$ at $240 \mathrm{~km}$ using the single-stage linearization filter.

from the signal to partially compensate the SSBI. The advantage of this technique is its use of a very simple DSP structure. However, as the calculation of signal-signal beating products is based on the received distorted signal, this technique itself introduces extra beating interference, thus limiting the compensation performance. In back-to-back operation, as shown in Fig. 3, the system performance was improved by using the single-stage linearization filter, with the required OSNR at the HD-FEC threshold reducing to $27.8 \mathrm{~dB}$ (a $5.1 \mathrm{~dB}$ gain). The performance difference between Rx- and Tx-EDC schemes combined with single-stage linearization filter is shown in Fig. 6. It can be observed that the BER was reduced and also the performance difference between Rx-EDC and Tx-EDC was reduced. Fig. 6(a) shows that from $80 \mathrm{~km}$ to $240 \mathrm{~km}$, BER ranged from $1.9 \times 10^{-4}$ to $4.0 \times 10^{-3}$ for Rx-EDC and from $7.5 \times 10^{-5}$ to $2.1 \times 10^{-3}$ for Tx-EDC, BERs being approximately halved with the use of Tx-EDC. Furthermore, the transmission performance of both EDC schemes over a range of launch powers was also improved, as plotted in Fig. 6(b).

\section{With Two-Stage Linearization Filter}

The Rx DSP design using the two-stage linearization filter followed by Rx-EDC is shown in Fig. 7. In the two-stage linearization filter [12], a second linearization stage is applied to remove the majority of the unwanted beating interference (mainly signal-SSBI beating products) introduced by the first linearization stage, the latter being identical to the above-mentioned single-stage linearization filter. Consequently, the second stage further enhances the compensation gain. As can be observed in Fig. 3, the system's back-to-back performance was further improved compared to the case with the single-stage linearization filter, the required OSNR value at HD-FEC threshold being reduced to $25.4 \mathrm{~dB}$ (a $7.5 \mathrm{~dB}$ gain) using the two-stage linearization filter. Furthermore, a comparison between both EDC

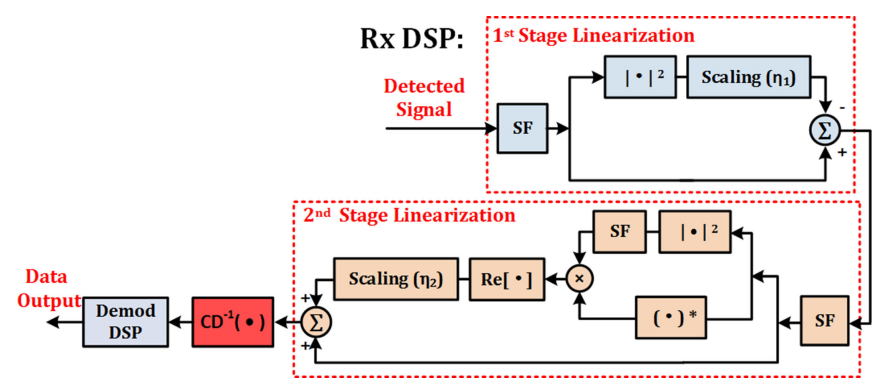

Fig. 7. Receiver DSP using the two-stage linearization filter and Rx-EDC. Demod DSP: Conventional demodulation DSP for SSB Nyquist-SCM signal. SF: Sideband filter (after [20]).

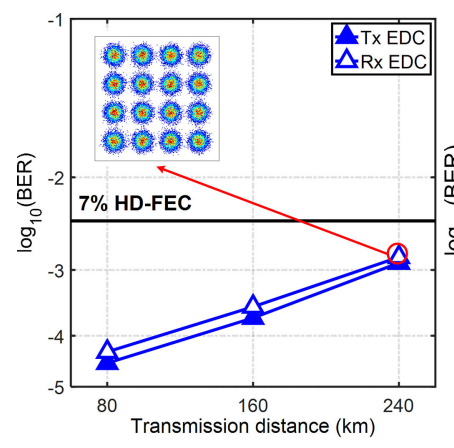

(a)

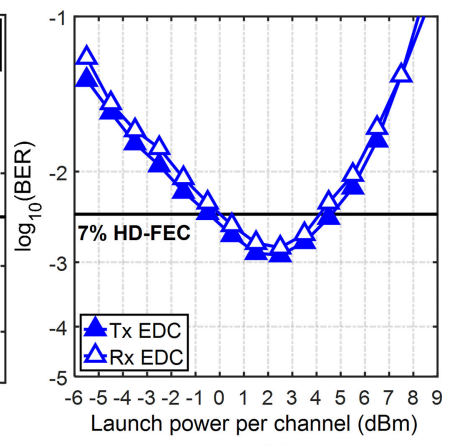

(b)
Fig. 8. (a) BER vs transmission distance with Tx-EDC and Rx-EDC using the two-stage linearization filter. Inset: Received constellation with Rx-EDC (EVM $=15.7 \%$ ) (after [20]). (b) BER vs launch power per channel with Tx-EDC and $\mathrm{Rx}-\mathrm{EDC}$ at $240 \mathrm{~km}$ using the two-stage linearization filter.

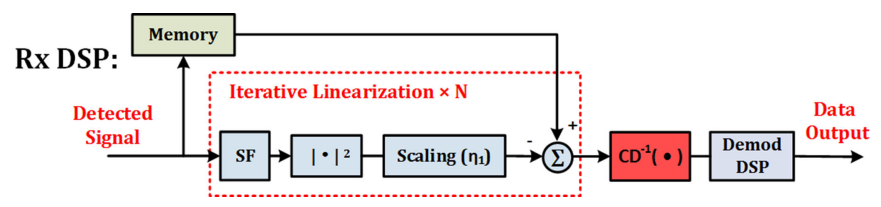

Fig. 9. Receiver DSP using the iterative linearization filter followed by the Rx-EDC. Demod DSP: Conventional demodulation DSP for SSB Nyquist-SCM signal. SF: Sideband filter.

schemes utilizing the two-stage linearization filter is shown in Fig. 8. Further improvement of BERs was achieved and RxEDC and Tx-EDC showed very similar performance. As can be seen from Fig. 8(a), from $80 \mathrm{~km}$ to $240 \mathrm{~km}$, the BER ranged from $4.8 \times 10^{-5}$ to $1.5 \times 10^{-3}$ when using Rx-EDC and from $3.4 \times 10^{-5}$ to $1.2 \times 10^{-3}$ with Tx-EDC, which is marginally lower. The slight difference in obtained BER values shown in Fig. 8(a) and (b) is mainly due to the residual uncompensated beating terms introduced by the second-stage of the linearizing filter.

\section{With Iterative Linearization Filter}

Fig. 9 shows the Rx DSP design using the iterative linearization filter and Rx-EDC. The iterative linearization filter [5] is an alternative method to further improve the performance of the single-stage linearization filter: the detected signal waveform is first stored in memory, the signal-signal beating products are calculated based on the sideband-filtered signal (in the same way as with the single-stage linearization filter described in Section III-B), which are then subtracted from the stored signal waveform in the memory to mitigate the SSBI. This process is 


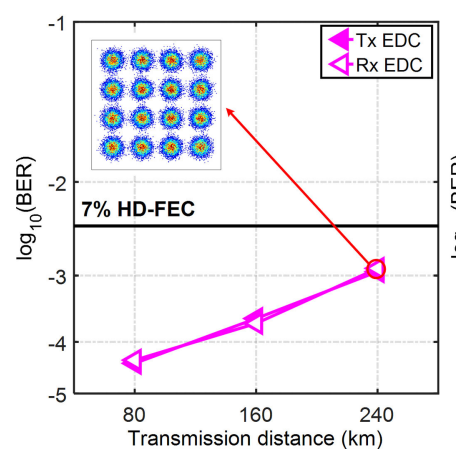

(a)

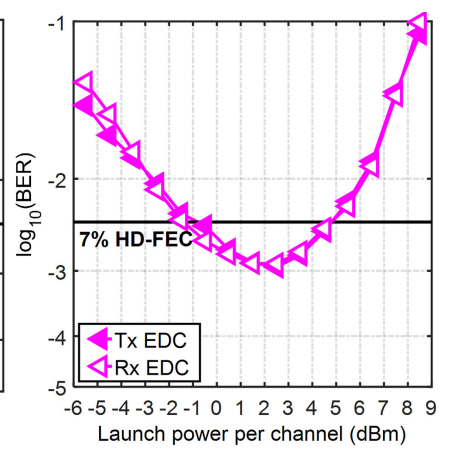

(b)
Fig. 10. (a) BER vs transmission distance with Tx-EDC and Rx-EDC using the iterative linearization filter. Inset: Received constellation with Rx-EDC $(\mathrm{EVM}=15.3 \%)$. (b) BER vs launch power per channel with Tx-EDC and $\mathrm{Rx}-\mathrm{EDC}$ at $240 \mathrm{~km}$ using the iterative linearization filter.

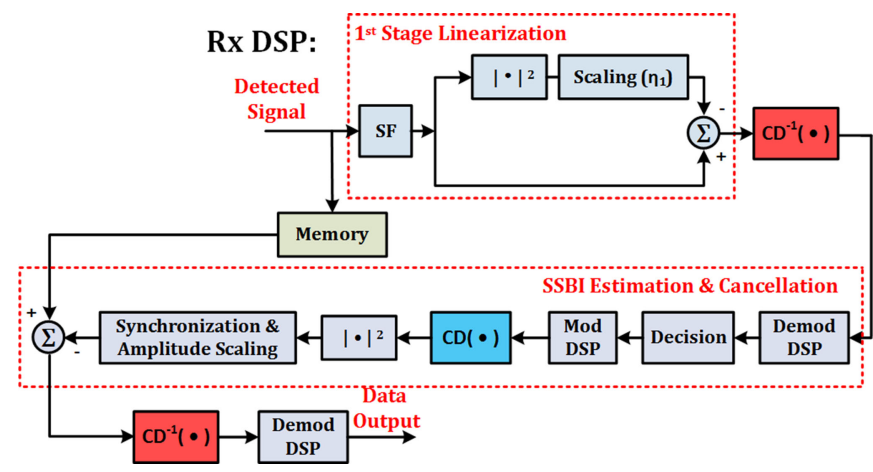

Fig. 11. Receiver DSP using the SSBI estimation and cancellation and RxEDC. Mod \& Demod DSP: Conventional modulation and demodulation DSP for SSB Nyquist-SCM signal. SF: Sideband filter.

repeated multiple times in order to achieve the maximum compensation gain. However, while improving the compensation performance, this comes at the expense of a significant increase in DSP complexity due to the multiple (four or more) iterations that need to be performed. The back-to-back assessment shown in Fig. 3 indicates that the iterative linearization filter also improved on the performance of the single-stage linearization filter, the required OSNR at HD-FEC threshold being reduced to $25.7 \mathrm{~dB}$ using the iterative linearization filter (a 7.2 $\mathrm{dB}$ gain). Moreover, as shown in Fig. 10, for the case of the iterative linearization filter, Rx-EDC and Tx-EDC achieved almost identical performance. Fig. 10(a) shows that from $80 \mathrm{~km}$ to $240 \mathrm{~km}$, the BER ranged from $4.7 \times 10^{-5}$ to $1.2 \times 10^{-3}$ for $\mathrm{Rx}-\mathrm{EDC}$ and from $4.1 \times 10^{-5}$ to $1.1 \times 10^{-3}$ for Tx-EDC. Fig. 10(b) also indicates that both EDC schemes exhibit the same performance at different launch powers. It can also be observed that the transmission performance of Rx-EDC combined with the iterative linearization filter is slightly better than that achieved through the use of a two-stage linearization filter (Section IIIC). However, since five iterations were required to achieve the maximum compensation gain in the experiment, the required DSP complexity is much higher than the single- and two-stage linearization filters techniques.

\section{E. With SSBI Estimation and Cancellation Technique}

The Rx DSP including the SSBI estimation and cancellation technique and Rx-EDC is shown in Fig. 11. The SSBI estima-

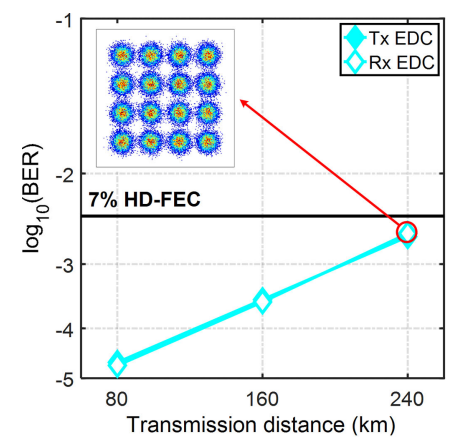

(a)

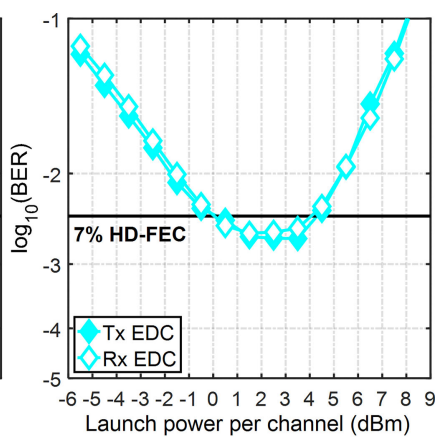

(b)
Fig. 12. (a) BER vs transmission distance with Tx-EDC and Rx-EDC using the SSBI estimation and cancellation. Inset: Received constellation with Rx$\mathrm{EDC}(\mathrm{EVM}=17.2 \%)$. (b) BER vs launch power per channel at $240 \mathrm{~km}$ with Tx-EDC and Rx-EDC with SSBI estimation and cancellation.

tion and cancellation technique [13] is an updated version of the previously proposed iterative SSBI estimation and cancellation scheme [14], [15]; it offers compensation performance similar to the iterative SSBI estimation and cancellation technique and avoids the requirement for multiple (typically three or four) symbol decision-based SSBI reconstruction processes, which requires multiple IFFT/FFT pairs. In the SSBI estimation and cancellation scheme, two copies of the detected signal (which is double sideband following detection) are made with one being stored in memory and the other being passed through the single-stage linearization filter (as described in Section III-B) to partially eliminate the SSBI terms. Following this, non-iterative SSBI estimation and cancellation is performed: a representation of the SSB SCM signal is generated by modulation DSP (Mod DSP) and an approximation of the signal-signal beating products is constructed and then subtracted from the stored signal waveform. Unlike the linearization filtering schemes, this technique does not introduce additional unwanted beating products, and thus it offers potentially better compensation gain especially at higher OSNR levels. However, the limitation of this technique is its dependency on the accuracy of the symbol decision making, which noticeably degrades its performance at lower OSNR values. In the measurement of this scheme's backto-back performance (Fig. 3), the required OSNR was $25.9 \mathrm{~dB}$ (a $7.0 \mathrm{~dB}$ gain). Following this, performance comparisons between both EDC schemes with the SSBI estimation and cancellation technique were carried out (Fig. 12). Again, very similar performance can be observed. Fig. 12(a) shows that from $80 \mathrm{~km}$ to $240 \mathrm{~km}$, BER ranged from $1.9 \times 10^{-5}$ to $2.4 \times 10^{-3}$ for Rx-EDC and from $2.2 \times 10^{-5}$ to $2.2 \times 10^{-3}$ for Tx-EDC. Similar performance was also observed over a range of optical launch powers at $240 \mathrm{~km}$ transmission, as shown in Fig. 12(b).

\section{F. With Kramers-Kronig Algorithm}

Following the assessment of SSBI mitigation schemes, we tested the performance of the recently-proposed KramersKronig scheme [11], [19] in the experiment. If the transmitted signal is minimum phase, the KK scheme enables the optical phase to be reconstructed digitally from the measurement of the optical signal's envelope (making the assumption that the optical signal is single-sideband). This scheme has been predicted to have outstanding performance in simulations [11]. In order to fulfill the condition of minimum phase, the optical carrier is required to have an amplitude larger than that of the signal. Fig. 13 


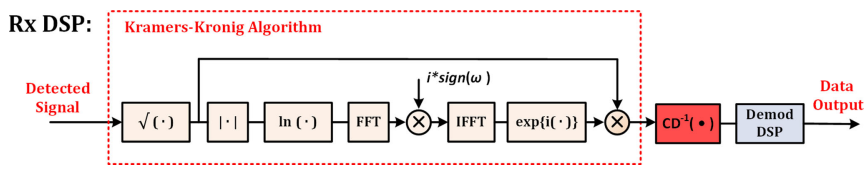

Fig. 13. Receiver DSP using the Kramers-Kronig algorithm and Rx-EDC. Mod \& Demod DSP: Conventional modulation and demodulation DSP for SSB Nyquist-SCM signal. SF: Sideband filter.

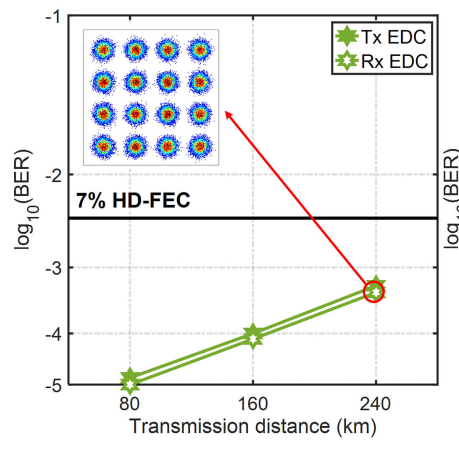

(a)

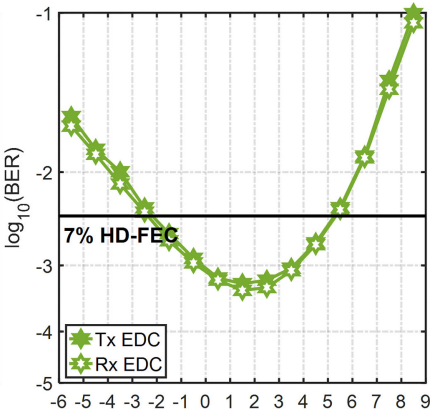

Launch power per channel $(\mathrm{dBm})$

(b)
Fig. 14. (a) BER vs transmission distance with Tx-EDC and Rx-EDC using the Kramers-Kronig algorithm. Inset: Received constellation with Rx-EDC $(\mathrm{EVM}=13.2 \%)$. (b) BER vs launch power per channel at $240 \mathrm{~km}$ with Tx-EDC and Rx-EDC with KK scheme.

shows the Rx DSP including the Kramers-Kronig algorithm and Rx-EDC. Based on the Kramers-Kronig relation, the phase of the transmitted signal is linked to its intensity. Hence, following direct detection of the total field intensity, the complex-valued electric field of the SSB signal is extracted from the measured photocurrent. The KK algorithm being utilized to recover the complex waveform of the optical signal can be written as:

$$
\begin{aligned}
h(n) & =\operatorname{sqrt}\left(V_{D D}(n)\right) . \\
\varphi(n) & =\mathcal{F}^{-1}\{i \operatorname{sign}(\omega) \mathcal{F}\{\ln [|h(n)|]\}\} . \\
V_{K K}(n) & =h(n) \cdot \exp \{i \varphi(n)\} .
\end{aligned}
$$

where $\operatorname{VDD}(n)$ is the $\mathrm{DSB}$ real-valued signal obtained after direct detection of the optical SSB signal, $n$ is the discrete time index, $\operatorname{sign}(\omega)$ is the sign function, which is equal to 1 for $\omega>0$, to 0 for $\omega=0$, and to -1 for $\omega<0$, and $F^{-1}\{\bullet\}$ and $F\{\bullet\}$ are the inverse Fourier and Fourier transform operators.

Note that, due to the high bandwidth resulting from the square-root and logarithm $(\ln (|h(n)|))$ operations, a relatively higher oversampling rate (4 samples per symbol) was used in this part of the DSP.

As shown in Fig. 3, it can be seen that the KK algorithm provided the best performance of all the schemes in back-toback operation and the required OSNR was only $23.5 \mathrm{~dB}(9.4 \mathrm{~dB}$ gain). Furthermore, as shown in Fig. 14, when the KK algorithm was applied in transmission, Tx- and Rx-EDC schemes achieved the same performance while, at the same the time, the achieved BER was lower than for all other linearization schemes. As shown in Fig. 14(a) and (b), the BER ranged from $1.0 \times 10^{-5}$ to $3.6 \times 10^{-4}$ for Rx-EDC and from $1.4 \times 10^{-5}$ to $4.0 \times 10^{-4}$ for Tx-EDC, and both EDC schemes offer the same performance over the investigated range of launch powers.

\section{G. Comparison of the Different Schemes}

From Fig. 3, it can be observed that the system's back-to-back performance was significantly improved when the linearization

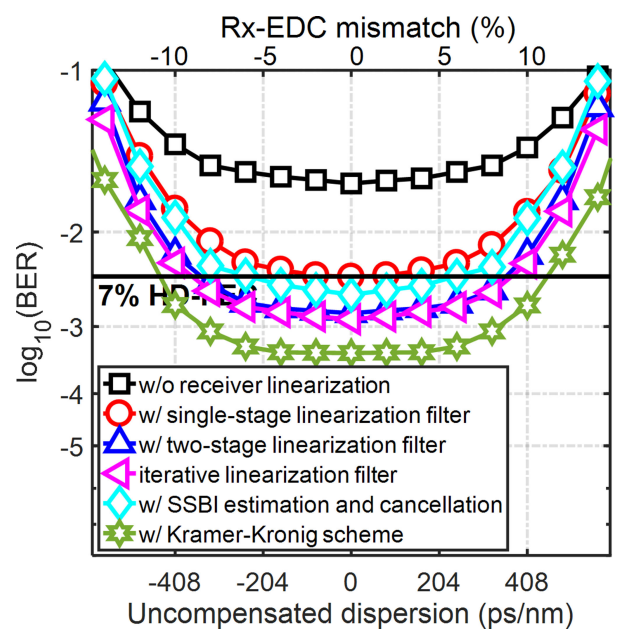

Fig. 15. BER versus uncompensated dispersion in case of without beating interference compensation, and by applying different linearization techniques after $240 \mathrm{~km}$ transmission at the $2.5 \mathrm{dBm}$ optical launch power per channel.

methods were performed. The performance of the single-stage linearization filter can be further improved by adding an extra linearization stage (two-stage linearization) or repeating this process by multiple (five or more) times. The SSBI estimation and cancellation scheme provides better compensation performance at high OSNRs, although its performance is noticeably degraded at lower OSNR levels due to increased number of inaccurate symbol decisions [18]. In order to achieve the maximum compensation gain, it is also necessary to optimize the amplitude scaling factor utilized in each linearization scheme, in schemes with one scaling factor (single-stage and iterative linearization filters), the scaling factor is optimized by sweeping its value and selecting the optimum value achieving the minimum BER. With the two-stage linearization filter and the SSBI estimation and cancellation schemes, both of which contain two scaling factors, the scaling factor in the first linearization stage is initially optimized (with the second linearization stage switched off), and the second scaling factor is optimized with the first scaling factor set to the optimum value [12]. Moreover, since linearization techniques change the tradeoff between the nonlinear and linear beating terms, the carrier-to-signal power ratio needs to be optimized to different values for the optimum performance. In contrast to the case without beating interference compensation, due to their different capabilities in suppressing the SSBI, the optimum CSPR is reduced by $2 \mathrm{~dB}$ for the single-stage linearization filter, $3 \mathrm{~dB}$ for the iterative, two-stage linearization filters and SSBI estimation and cancellation technique and $5 \mathrm{~dB}$ for the KK scheme for all values of OSNR.

In order to observe the sensitivity of the received signal quality towards variations of the fiber chromatic dispersion for different linearization techniques, the measured BER versus uncompensated dispersion (or Rx-EDC mismatch) after $240 \mathrm{~km}$ transmission at $2.5 \mathrm{dBm}$ optical launch power per channel is plotted in Fig. 15. It can be seen that the different linearization techniques exhibit similar tolerance to dispersion variation.

Finally, all four WDM channels were tested with Rx-EDC over a transmission distance of $240 \mathrm{~km}$, with similar performance across all channels being observed (Fig. 16). Assuming a hard-decision forward error correction (HD-FEC) overhead of $7 \%$, allowing a pre-FEC BER $=3.8 \times 10^{-3}$, the net information spectral density of the WDM signal is calculated to be $2.8 \mathrm{~b} / \mathrm{s} / \mathrm{Hz}$ 


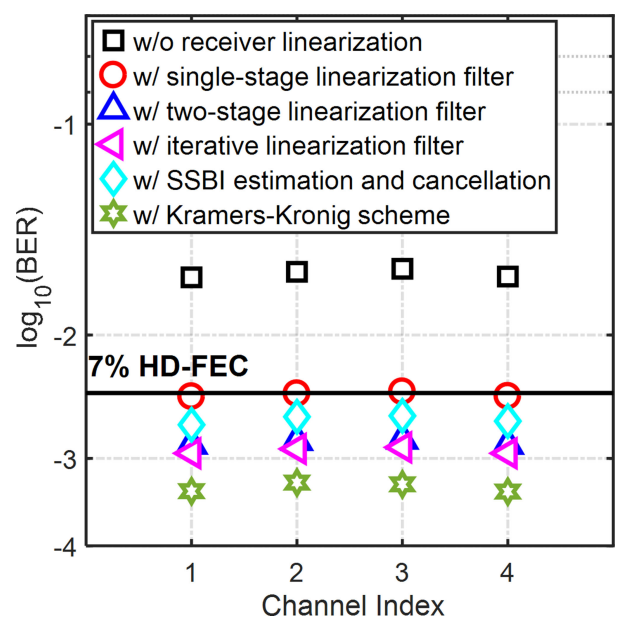

Fig. 16. BER versus WDM channel index with Rx-EDC in case of without beating interference compensation, and by applying different linearization techniques after $240 \mathrm{~km}$ transmission.

In transmission, the optimum CSPR values were chosen to be $14 \mathrm{~dB}$ for $80 \mathrm{~km}(\mathrm{OSNR}=37 \mathrm{~dB})$ and $11 \mathrm{~dB}$ for $240 \mathrm{~km}$ $($ OSNR $=32 \mathrm{~dB})$ transmissions, reducing by $2 \mathrm{~dB}$ to $12 \mathrm{~dB}$ and $9 \mathrm{~dB}$ with single-stage linearization filter, and it was further reduced by $3 \mathrm{~dB}$ to $11 \mathrm{~dB}$ and $8 \mathrm{~dB}$ with two-stage, iterative linearization filters and with SSBI estimation and cancellation technique. For the case with KK algorithm, due to its superior linearization performance, a reduction of optimum CSPR of up to $5 \mathrm{~dB}$ was observed, the optimum CSPR decreasing to just $9 \mathrm{~dB}$ and $6 \mathrm{~dB}$ for $80 \mathrm{~km}$ and $240 \mathrm{~km}$ transmissions respectively.

\section{CONCLUSiOnS}

We reported an experimental study on the effectiveness of performing electronic dispersion compensation (EDC) either at the transmitter (Tx-EDC) or at the receiver ( $\mathrm{Rx}$-EDC) in directdetection (DD) subcarrier modulation (SCM) transceivers combined with different receiver linearization techniques. Five different recently proposed linearization techniques including a single-stage linearization filter, a two-stage linearization filter, an iterative linearization filter, an SSBI estimation and cancellation technique and the Kramers-Kronig scheme were assessed. The performance of both EDC schemes was experimentally evaluated in a $4 \times 112 \mathrm{~Gb} / \mathrm{s}$ spectrally-efficient $(2.8 \mathrm{~b} / \mathrm{s} / \mathrm{Hz}$ net ISD) WDM direct-detection single-sideband 16-QAM NyquistSCM system over reaches of up to $240 \mathrm{~km}$ of uncompensated SSMF, and was compared with the performance of transmitterbased electronic dispersion compensation (Tx-EDC). The experimental results indicate that the performance difference of Tx and Rx-EDC depends on the effectiveness of the linearization scheme that is used, and that they can achieve similar performance if the beating interference is effectively suppressed. Therefore, it becomes possible to perform the EDC at the receiver rather than at the transmitter, which simplifies the system operation since knowledge of link dispersion is not required at the transmitter. Due to the reduction in complexity, the proposed solution increases the suitability of WDM DD SSB SCM signaling for short- and medium-reach applications such as metro networks, back-haul, access and inter-data center links. To the best of our knowledge, this study included the first experimental evaluation of the Kramers-Kronig (KK) scheme in DD transmis- sion system, the results showing that the KK scheme not only enables both EDC schemes to exhibit similar performance, but also provides superior linearization effectiveness over the other beating interference mitigation schemes being compared.

\section{REFERENCES}

[1] A. O. Wiberg, B.-E. Olsson, and P. A. Andrekson, "Single cycle subcarrier modulation," in Proc. Opt. Fiber Commun., 2009, Paper OTuE1.

[2] J. C. Cartledge and A. S. Karar, " $100 \mathrm{~Gb} / \mathrm{s}$ intensity modulation and direct detection," J. Lightw. Technol., vol. 32, no. 16, pp. 2809-2814, Aug. 2014

[3] K. Zhong et al., "Experimental demonstration of 608Gbit/s short reach transmission employing half-cycle 16QAM Nyquist-SCM signal and direct detection with 25 Gbps EML," Opt. Express, vol. 24, no. 22, pp. 25057-25067, 2016.

[4] M. S. Erkılınç et al., "Spectrally-efficient WDM Nyquist-pulse-shaped 16-QAM subcarrier modulation transmission with direct detection," $J$. Lightw. Technol., vol. 33, no. 15, pp. 3147-3155, Aug. 2015.

[5] K. Zou, Y. Zhu, F. Zhang, and Z. Chen, "Spectrally efficient terabit optical transmission with Nyquist 64-QAM half-cycle subcarrier modulation and direct-detection," Opt. Lett., vol. 41, no. 12, pp. 2767-2770, 2016.

[6] R. I. Killey, P. M. Watts, V. Mikhailov, M. Glick, and P. Bayvel, "Electronic dispersion compensation by signal predistortion using digital processing and a dual-drive Mach-Zehnder modulator," IEEE Photon. Technol. Lett., vol. 17, no. 3, pp. 714-716, Mar. 2005.

[7] M. Sieben, J. Conradi, and D. E. Dodds, "Optical single sideband transmission at $10 \mathrm{~Gb} / \mathrm{s}$ using only electrical dispersion compensation," J. Lightw. Technol., vol. 17, no. 10, pp. 1742-1749, Oct. 1999.

[8] Q. Zhang et al., "C-band 56Gbps transmission over 80-km single mode fiber without chromatic dispersion compensation by using intensitymodulation direct-detection," in Proc. Eur. Conf. Opt. Commun., 2014, Paper P.5.19.

[9] L. Zhang et al., "Beyond 100-Gb/s transmission over 80-km SMF using direct-detection SSB-DMT at C-band," J. Lightw. Technol., vol. 34, no. 2, pp. 723-729, Jan. 2016.

[10] S. Randel, D. Pilori, S. Chandrasekhar, G. Raybon, and P. Winzer, " $100-\mathrm{Gb} / \mathrm{s}$ discrete-multitone transmission over $80-\mathrm{km}$ SSMF using single-sideband modulation with novel interference-cancellation scheme," in Proc. Eur. Conf. Opt. Commun., 2015, Paper Mo.4.5.2

[11] A. Mecozzi, C. Antonelli, and M. Shtaif, "Kramers-Kronig coherent receiver," Optica, vol. 3, no. 11, pp. 1220-1227, 2016.

[12] Z. Li et al., "Two-stage linearization filter for direct-detection subcarrier modulation," IEEE Photon. Technol. Lett., vol. 28, no. 24, pp. 2838-2841, Dec. 2016.

[13] Z. Li et al., "Reach enhancement for WDM direct-detection subcarrier modulation using low-complexity two-stage signal-signal beat interference cancellation," in Proc. Eur. Conf. Opt. Commun., 2016, Paper M.2.B.1.

[14] W. R. Peng et al., "Spectrally efficient direct-detected OFDM transmission employing an iterative estimation and cancellation technique," Opt. Express, vol. 17, no. 11, pp. 9099-9111, 2009.

[15] Z. Li et al., "Signal-signal beat interference cancellation in spectrallyefficient WDM direct-detection Nyquist-pulse-shaped 16-QAM subcarrier modulation," Opt. Express, vol. 23, no. 18, pp. 23694-23709, 2015.

[16] C. Sánchez, B. Ortega, and J. Capmany, "System performance enhancement with pre-distorted OOFDM signal waveforms in IM/DD systems," Opt. Express, vol. 22, no. 6, pp. 7269-7283, 2014.

[17] C. Ju, N. Liu, X. Chen, and Z. Zhang, "SSBI mitigation in a RF-tone-based VSSB-OFDM system with a frequency-domain Volterra series equalizer," J. Lightw. Technol., vol. 33, no. 23, pp. 4997-5006, Dec. 2015.

[18] Z. Li et al., "Comparison of digital signal-signal beat interference compensation techniques in direct-detection subcarrier modulation systems," Opt. Express, vol. 24, no. 25, pp. 29176-29189, 2016.

[19] H. Voelcker, "Demodulation of single-sideband signals via envelope detection," IEEE Trans. Commun. Technol., vol. COM-14, no. 1, pp. 22-30, Feb. 1966

[20] Z. Li et al., "Performance improvement of electronic dispersion postcompensation in direct detection systems using DSP-based receiver linearization," in Proc. Opt. Fiber Commun., 2017, Paper Th3D.2.

[21] H. Bülow, F. Buchali, and A. Klekamp, "Electronic dispersion compensation," J. Lightw. Technol., vol. 26, no. 1, pp. 158-167, Jan. 2008.

Authors' biographies not available at the time of publication. 